\title{
El efecto de la estructura de las redes sociales sobre la confianza. Un análisis de simulaciones computarizadas y evaluación de la tesis de Coleman
}

\author{
José Ignacio García-Valdecasas \\ Universidad de Valladolid. Departamento de Sociología y Trabajo Social \\ joseignacio.garcia-valdecasas@uva.es
}

Recepción: 14-02-2018

Aceptación: 14-11-2018

Publicación: 26-07-2019

\section{Resumen}

El objetivo del artículo es analizar el impacto de distintos tipos de estructuras y de diferentes propiedades estructurales de las redes sociales sobre la tendencia a confiar en el interior de ellas. Para llevar a cabo dicho objetivo, se han realizado una serie de experimentos virtuales con redes artificiales a través de técnicas de simulación computarizadas. Los resultados ponen de manifiesto el profundo efecto de las propiedades estructurales (densidad de red, índice de globalización de vínculos e índice de Gini de la distribución de vínculos) y del tipo de estructura (regular, aleatoria y mundo pequeño) de las redes sociales sobre la tendencia a confiar entre los agentes de una red. Además, se evalúa la tesis de Coleman sobre el control y la vigilancia de los jóvenes para no abandonar los estudios a partir de los datos suministrados por los experimentos virtuales realizados con las redes artificiales anteriores.

Palabras clave: mundo pequeño; coeficiente de conglomerado; índice de Gini; experimentos virtuales; redes artificiales; simulación computarizada; tesis de Coleman

\begin{abstract}
The effect of social network structures on trust: An analysis based on computer simulations and the evaluation of Coleman's theory
\end{abstract}

The aim of this article is to analyze the impact of different social network structures and structural properties on the tendency to trust inside networks. To this end, a series of virtual experiments with artificial networks have been carried out using computer simulations. The results underscore the profound effect of the structural properties (network density, link globalization index and Gini index of the distribution of links) and type of structure (regular, random and small world) of social networks on the tendency to trust among the agents of a network. In addition, Coleman's theory on the control and surveillance of young people is evaluated to study the data provided by the virtual experiments with the previous artificial networks.

Keywords: small world; clustering coefficient; Gini Index; virtual experiments; artificial networks; computer simulation; Coleman's theory 


\author{
Sumario \\ 1. Introducción 4. Experimentos virtuales \\ 2. Redes sociales y confianza \\ 5. Discusión y conclusiones \\ 3. Redes artificiales \\ Referencias bibliográfícas
}

\title{
1. Introducción
}

La confianza ha sido uno de los principales objetos de estudio de las ciencias sociales durante las últimas décadas. Así lo atestigua la multitud de enfoques teóricos y la enorme cantidad de investigaciones empíricas que se han realizado sobre ella (Hardin, 2006; Rousseau et al., 1998). Se pueden esgrimir varios argumentos para defender el interés de las ciencias sociales por la confianza (Sztompka, 2006). En primer lugar, el centro de atención de las ciencias sociales se ha ido desplazando desde el nivel macro (estructuras, instituciones, funciones, etc.) hasta el nivel micro de los fenómenos sociales (interacciones, vínculos, etc.), y la confianza surge precisamente en los procesos micro de la vida social. En segundo lugar, el proceso de globalización, el fenómeno de las redes sociales digitales, así como el rápido cambio social que experimenta la sociedad actual conllevan la creación de vínculos distantes entre individuos desconocidos, y la existencia de dichos vínculos supone cierta incertidumbre a la hora de confiar en otros. Los individuos probablemente confíen menos en otros individuos en contextos de alta incertidumbre (Cook y Gerbasi, 2009). Además, la confianza puede desempeñar un papel crucial en muchas facetas de la vida económica, política y social. En la esfera económica, por ejemplo, si una persona compra un coche usado tiene que confiar en el vendedor cuando le pida una determinada cantidad de dinero por él, porque el comprador no tiene suficiente capacidad para juzgar con exactitud la calidad de dicho vehículo. Si una persona le presta dinero a un amigo confía en que su amigo no huya con el dinero y espera que se lo devuelva tan pronto como le sea posible (véanse más ejemplos en Snijders, 1996). Los comportamientos oportunistas de los vendedores y los potenciales daños para los compradores se pueden reducir o eliminar mediante contratos legales, pero dichos contratos suelen ser caros y, a veces, imposibles de llevar a cabo (Buskens, 1998: 266). Así pues, la confianza puede reducir los costes de los intercambios económicos y, por tanto, favorecer el desarrollo económico (Fukuyama, 1995). Asimismo, en la esfera política, la confianza puede estimular el progreso de la democracia, porque sin confianza es difícil que exista la democracia (Paramio, 1999; Putnam, 1993, 1995, 2000). Además, la confianza es crucial para la participación política y el desarrollo de la ciudadanía (Montero et al., 2008). En el mismo sentido, el filósofo político John Dunn (1993: 641) afirmaba: «La confianza mutua se encuentra en el núcleo de todos los procesos políticos». Por último, en la esfera social, la confianza entre los miembros que constituyen una organización, por ejemplo, es un factor clave para que esta logre sus objetivos y alcancen el éxito (Gasalla y Navarro, 2008; Lucas, 2013). 
Por otra parte, la relevancia de las estructuras de las redes sociales como factores explicativos de numerosos fenómenos sociales ha sido defendida por varios autores durante las últimas décadas (Boccaletti et al., 2006; Burt, 2005; Hedström, 2005; Hedström y Bearman, 2009). Por ejemplo, el mismo conjunto de individuos distribuidos de manera distinta en una red puede generar diferentes dinámicas sociales, como muestran diversos estudios pioneros en el campo de las redes sociales (Coleman et al., 1957; Granovetter, 1973; Milgram, 1967; Schelling, 1978). Incluso pequeñas modificaciones en la estructura de interacción entre los individuos pueden tener un gran impacto en los resultados sociales, como ponen de manifiesto las investigaciones realizadas por autores procedentes de otros campos distintos de los de las ciencias sociales (Barabási, 2002; Newman et al., 2003; Watts, 2003). Así pues, no resulta extraño que se pueda afirmar que el análisis de las relaciones entre la estructura de las redes sociales (una manera de conceptualizar la estructura social) y los procesos dinámicos que tienen lugar en su interior (el comportamiento de los actores) sea uno de los campos más prometedores de las ciencias sociales de hoy en día (García-Valdecasas, 2015; Steglich et al., 2010).

Asimismo, se puede distinguir entre confianza generalizada y confianza relacional (Hardin, 2006). Mientras la confianza generalizada es la que se refiere a desconocidos sobre los que se carece de información acerca de si son o no dignos de confianza (Herreros, 2003), la confianza relacional es la que se refiere a conocidos sobre los que sí se dispone de información acerca de si son o no dignos de confianza. Por otro lado, algunos autores también distinguen, dentro de la confianza relacional, entre confianza vertical y horizontal (Sztompka, 2006). La confianza vertical se refiere, por ejemplo, a la confianza hacia las instituciones del Estado, mientras que la confianza horizontal se centra en la confianza entre los individuos que están conectados a través de una red. La lógica y la dinámica de ambas confianzas son completamente distintas. Este trabajo analiza la confianza horizontal, y dentro de la confianza horizontal se centra en la tendencia a confiar dentro de la red. Por último, se puede señalar que la confianza entre los miembros de una red está estrechamente relacionada con la cooperación entre dichos miembros (Axelrod, 1984; Back y Flache, 2006). Desde este punto de vista, la confianza es condición necesaria, aunque insuficiente para cooperar. Asimismo, la cooperación entre los miembros de una red es crucial para alcanzar los posibles objetivos comunes de dicha red.

Aunque existen diversos estudios acerca de qué modo las redes sociales influyen en la confianza generalizada (Chow y Chan, 2008; Wu y Chiclana, 2014), apenas existen trabajos que analicen cómo las estructuras de las redes sociales influyen sobre la tendencia a confiar (una notable excepción son los trabajos de Buskens, 1998, 2002; Burt, 2005; Burt et al., 2018). Otra excepción en contextos de las redes sociales virtuales es el reciente estudio de Ghavipour y Meybodi (2018). La escasez de estas investigaciones se puede deber a la dificultad de obtener datos empíricos detallados sobre la estructura de las redes sociales para estudiar dicho efecto. Para tratar de paliar esta falta de datos, el presente artículo usa técnicas de simulación computarizadas (Axelrod, 1997; Epstein, 
2006; García-Valdecasas; 2011, 2016; Gilbert, 2008; Gilbert y Abbott, 2005; Gilbert y Troitzsch, 2005; González-Bailón, 2004, 2006; Macy y Flache, 2009; Macy y Miller, 2002; Squazzoni, 2012), con el objetivo de realizar experimentos virtuales con redes artificiales para analizar el impacto de las estructuras de las redes sociales sobre la tendencia a confiar. Aunque la aplicación de las técnicas de simulación computarizadas para analizar las redes sociales es bastante novedosa, no es la primera vez que se utilizan para dicho propósito (véase, por ejemplo, los trabajos de Back y Flache, 2006 y Siegel, 2009).

Es necesario realizar algunas consideraciones generales sobre la simulación computarizada en la investigación social. Los experimentos virtuales no pueden suministrar - obviamente - datos empíricos y, por tanto, no pueden suplantar a la investigación empírica. No obstante, los resultados de dichos experimentos pueden proporcionar diversas hipótesis empíricas que se pueden comprobar posteriormente, así como plantear numerosos problemas teóricos que pueden enriquecer nuestra perspectiva del fenómeno social objeto de estudio (véase, por ejemplo, los trabajos de García-Valdecasas, 2013, 2014a; García-Valdecasas y López, 2017). Por tanto, la utilización de la simulación computarizada se puede emplear como guía de la investigación social de la misma forma que lo ha hecho tradicionalmente la teoría sociológica clásica. Además, los resultados de dichos experimentos también se pueden utilizar para valorar diversas tesis sociológicas. Este es uno de los usos más interesantes de la simulación computarizada (González-Bailón, 2006) y el que se utiliza en el presente trabajo.

Los fenómenos sociales están caracterizados, desde la perspectiva adoptada en este trabajo, por la creación, el mantenimiento y la ruptura de vínculos entre los agentes que constituyen dichos fenómenos sociales. Sin embargo, el objetivo del presente trabajo no se centra en la dinámica de un fenómeno social a lo largo del tiempo, sino que "congela» el fenómeno social para estudiar un aspecto estático. Así, esta es una investigación que analiza una red "fotografiada» en un momento determinado del tiempo.

De acuerdo con todo lo anterior, este artículo posee un doble objetivo: por una parte, analizar el efecto de diversas estructuras complejas de las redes sociales (regulares, aleatorias y mundos pequeños), así como de diferentes parámetros estructurales (densidad de red, índice de globalización de vínculos e índice de Gini de la distribución de vínculos) sobre la tendencia a confiar; es decir, trata de investigar cómo afecta un aspecto macro (la estructura de las redes y sus parámetros estructurales) a un aspecto micro (la tendencia a confiar). La relevancia de dichas estructuras complejas de las redes sociales radica en que están presentes en muchos tipos de redes (Watts, 2003), y la pertinencia de tales parámetros estructurales estriba en su enorme significado sociológico (García-Valdecasas, 2013, 2014a, 2015). Por otra parte, intenta mostrar la utilidad de la simulación computarizada con redes artificiales para valorar tesis sociológicas (González-Bailón, 2006). En este trabajo en particular se pretende evaluar la tesis de Coleman (1990) — a partir de los datos suministrados por los experimentos virtuales que se van a realizar- sobre la importancia de vigilar y controlar a los jóvenes para que no abandonen los estudios. La relación 
entre la vigilancia de los jóvenes y su asistencia a clase está mediada por las redes de interacción que pueden establecer con sus responsables, así como por la confianza que pueda surgir entre ellos. De aquí que se pueda evaluar dicha tesis con los resultados aportados por los experimentos con redes artificiales.

El artículo está organizado de la siguiente manera: primero, se analiza teóricamente la relación entre las redes sociales y la tendencia a confiar en el interior de dichas redes. Segundo, se describen las estructuras de las redes artificiales que se van a utilizar para llevar a cabo los experimentos virtuales y se ponen de manifiesto los mecanismos a través de los cuales se crean las redes. Tercero, se investiga el efecto de distintos tipos de estructuras de red, así como de diferentes propiedades estructurales sobre la tendencia a confiar. Por último, se utilizan los resultados de los experimentos virtuales para evaluar la tesis de Coleman (1990) sobre la vigilancia y el control de los jóvenes para que no dejen el colegio.

\section{Redes sociales y confianza}

Este apartado trata de la relación entre las redes sociales y la tendencia a confiar en el interior de dichas redes. Para ello se necesita definir en primer lugar qué son las redes sociales y qué es la tendencia a confiar. Posteriormente se explica la tesis de Coleman sobre la relación entre redes sociales y deserción escolar a través de la tendencia a confiar.

\subsection{Redes sociales}

Desde un punto de vista analítico, se puede definir una red social como un conjunto de actores o de agentes colectivos conectados entre sí a través de relaciones sociales. Los agentes pueden ser de diversas clases: personas, familias, empresas, estamentos sociales, naciones, etc., y las relaciones pueden ser también de distintos tipos: amistad, influencia, parentesco, sexualidad, vecindad, etc. (Molina, 2001; Requena, 1989; Rodríguez, 1995).

Asimismo, estas redes sociales se pueden representar a través de grafos o sociogramas (Moreno, 1934; Lewin, 1936, 1951), los cuales se pueden definir como un conjunto de nodos y un conjunto de vínculos que unen o que conectan pares de nodos. Es importante distinguir entre redes sociales y grafos: las redes sociales forman parte de la realidad social, pero los grafos son modelos de dicha realidad. Es decir, los grafos representan de manera simplificada a las redes sociales; los nodos simbolizan a los agentes, y los vínculos, a las relaciones. Además, los parámetros matemáticos de los grafos representan abreviadamente las propiedades estructurales de las redes sociales (Wasserman y Faust, 1994; Hanneman y Riddle, 2005). Evidentemente, la simplicidad de los grafos no puede agotar la complejidad ni la riqueza de las redes sociales, pero sin grafos somos incapaces de entender las redes sociales. En resumen, los grafos junto con los indicadores matemáticos de dichos grafos constituyen una «herramienta muy útil» para describir y explicar las redes sociales. 
Las propiedades estructurales de las redes sociales utilizadas en este trabajo son las siguientes: la densidad de red, el coeficiente de clustering medio de red (clustering es un término inglés que se puede traducir en español por 'conglomerado'), el índice de globalización de vínculos y el índice de Gini de la distribución de vínculos (véanse los trabajos de García-Valdecasas, 2013, 2014a, 2015). Todas estas propiedades son relevantes desde un punto de vista sociológico. Se definen ahora las dos primeras propiedades, y en el próximo apartado, las dos últimas.

La densidad de red $(D)$ es el cociente entre el número de vínculos existentes y el número de vínculos posibles de una red (Carrington et al., 2005; Diestel, 2000; Wasserman y Faust, 1994). Se puede calcular mediante la siguiente fórmula:

$$
D=\frac{2 m}{n(n-1)}
$$

siendo $m$ el número total de vínculos de la red, y $n$, el número total de nodos de dicha red. Así pues, la densidad mide el grado de conectividad de la red, y su valor varía entre 0 y 1 . Existen otras medidas de la conectividad de una red, pero la densidad es la más sencilla y resulta adecuada para los propósitos de este artículo. Además, cuanto mayor sea la densidad de una red, mayor será el costo que se necesite para la creación y el mantenimiento de dicha red (Wasserman y Faust, 1994; Hanneman y Riddle, 2005).

El coeficiente de conglomerado de un nodo $i\left(C_{i}\right)$ es el cociente entre el número de vínculos existente entre los nodos conectados al nodo $i$ y el número de vínculos posible entre dichos nodos. Mide, por tanto, el grado de conectividad entre los nodos conectados a un nodo $i$. El coeficiente de conglomerado medio de red $(\bar{C})$ es la media de los coeficientes de conglomerado de todos los nodos. Se puede calcular a través de la siguiente fórmula:

$$
\bar{C}=\frac{\sum_{i} C_{i}}{n}
$$

siendo $n$ el número total de nodos de dicha red.

Dicho coeficiente varía entre 0 y 1 . De esta definición se desprende que, a medida que aumenta la densidad de red, puede incrementarse el coeficiente de conglomerado medio de red.

En este trabajo también es útil distinguir entre redes cerradas y redes abiertas. Cuanto mayor es el coeficiente de conglomerado medio de una red, más cerrada es esta. Es decir, a medida que aumenta el número de vínculos entre los nodos conectados a cada uno de los nodos de una red, más cerrada es, y, por consiguiente, menos abierta. El valor del coeficiente de conglomerado de una 
Figura 1. Red completamente cerrada. $\bar{C}=1$

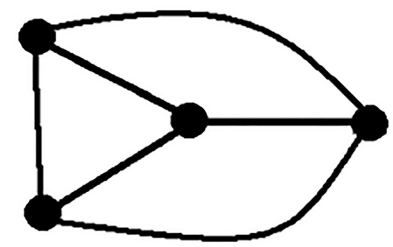

Fuente: elaboración propia

Figura 2. Red totalmente abierta. $\bar{C}=0$

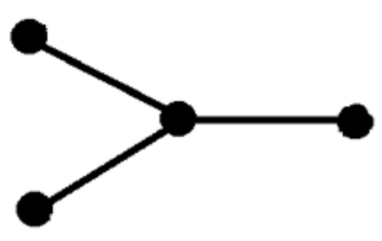

Fuente: elaboración propia

red completamente cerrada es 1 (figura 1 ), y el de una red totalmente abierta es 0 (figura 2). A las redes o subredes completamente cerradas se les denomina clique (término inglés) o camarilla (vocablo español).

\subsection{Tendencia a confiar}

Existen muchas definiciones de la palabra confianza, pero este artículo parte de la definición de Coleman (1990), porque permite relacionar la tendencia a confiar y la estructura de las redes sociales. La decisión de un individuo $i$ de confiar en otro individuo $j$ se puede considerar como una decisión racional (Alesina y La Ferrara, 2002; Coleman, 1990; García-Valdecasas, 2011 b; Herreros, 2002, 2004). Consideremos que $p_{i j}$ es la probabilidad subjetiva para el individuo $i$ de que el individuo $j$ sea digno de confianza, y $\left(1-p_{i j}\right)$ es la probabilidad subjetiva para el individuo $i$ de que el individuo $j$ no sea digno de confianza. Supongamos también que $G_{i}$ es la ganancia potencial para el individuo $i$ si el individuo $j$ honra la confianza depositada en él y que $R_{i}$ es la pérdida potencial para el individuo $i$ de que el individuo $j$ no honre dicha confianza. Consideremos ahora que el individuo $i$ es indiferente ante el riesgo que supone la decisión de confiar, esto es, que es completamente neutral frente a dicho riesgo. El individuo $i$ decidirá confiar en el individuo $j$ si:

$$
p_{i j} G_{i}>\left(1-p_{i j}\right) R_{i}, \quad \text { siendo } \quad p_{i j}[0,1], G_{i} \geq 0 \text { y } R_{i} \geq 0,
$$


por consiguiente:

$$
p_{i j} G_{i} /\left(1-p_{i j}\right) R_{i}>1
$$

Por tanto, el individuo $i$, que no posee ni atracción ni fobia por el riesgo, decidirá confiar en el individuo $j$ si la inecuación [1] se cumple. Así pues, la decisión del individuo $i$ de confiar en el individuo $j$ depende de tres factores: la probabilidad subjetiva de $i$ de confiar en $j\left(p_{i j}\right)$, las ganancias potenciales de $i$ si $j$ honra la confianza depositada en él $\left(G_{i}\right)$ y las pérdidas potenciales de $i$ si $j$ no honra dicha confianza $\left(R_{i}\right)$. Es importante notar que si la probabilidad subjetiva de $i$ de confiar en $j$ es muy baja, esto es, si $i$ confía muy poco en $j$, es posible, a pesar de todo, que $i$ decida confiar en $j$ si las ganancias potenciales de $i$ son muy elevadas frente a los costes potenciales (Herreros, 2002, 2004).

La decisión de confiar es dicotómica: sí o no. Si la inecuación [1] se cumple, entonces se toma la decisión de confiar, y si no se cumple, se toma la decisión de no confiar. Sin embargo, en esta investigación no se utiliza la decisión de confiar, sino la tendencia de un individuo $i$ a confiar en el individuo $j\left(T_{i j}\right)$. Dicha tendencia a confiar se puede concebir como la certidumbre en la decisión de confiar. Y se puede calcular mediante la siguiente fórmula:

$$
T_{i j}=p_{i j} G_{i} /\left(1-p_{i j}\right) R_{i}
$$

La tendencia a confiar es una función continua que varía entre 0 (cuando $p_{i j}=0$ o $\left.G_{i}=0\right)$ e $\infty$ (cuando $p_{i j}=1$ o $R_{i}=0$ ), excepto cuando $G_{i}=R_{i}=0$. Teniendo en cuenta la inecuación [1]: si $T_{i j}>1$, entonces se toma la decisión de confiar, pero si $T_{i j} \leq 1$, entonces se toma la decisión de no confiar, pero en este caso lo importante es la magnitud de la tendencia a confiar. Por ejemplo, si $T_{i j}=100$, entonces la certidumbre de la decisión de confiar es mayor que si $T_{i j}^{i j}=10$. Así pues, la tendencia a confiar puede ser concebida como una medida de la certidumbre con que se toma la decisión de confiar. Esta medida nos permite comparar diferentes tendencias a confiar entre distintos nodos.

Por último, se define la tendencia a confiar dentro de una red $(T)$ constituida por un conjunto $n$ de nodos mediante la siguiente fórmula:

$$
T=\frac{\sum_{i=1}^{n} \sum_{j=1}^{n} T_{i j}}{n(n-1)},
$$

siendo $i \neq j$ y $n$ el número total de nodos de una red.

Es importante también en este trabajo distinguir entre vínculos fuertes y débiles (Burt, 2004, 2005; Granovetter, 1973, 1974). Los individuos suelen estar rodeados por un número pequeño de personas con las que mantienen un contacto estrecho y frecuente, como la familia o los amigos, a través de vínculos fuertes. Junto a este núcleo fuerte existe un gran número de vínculos 
Figura 3. Vínculo débil

Fuente: elaboración propia

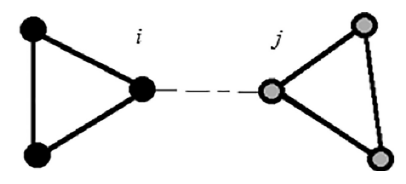

Figura 4. Vínculo fuerte

Fuente: elaboración propia

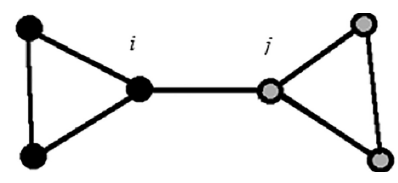

débiles con otros individuos con los que el contacto es más superficial y menos frecuente, como los conocidos, los vecinos o los colegas. Según Granovetter (1973), la fuerza de un vínculo es una combinación lineal de la cantidad de tiempo, la intensidad emocional, la ayuda recíproca y la intimidad mutua que caracteriza al vínculo (véase también el trabajo crítico de Cruz y Verd, 2013).

Supongamos ahora que dos individuos $i$ y $j$ crean un vínculo débil entre ellos por carecer de una historia común (la línea discontinua de la figura 3). La probabilidad subjetiva de confiar en el otro será menor que si tuvieran un vínculo fuerte basado en el conocimiento mutuo a través del tiempo (la línea continua de la figura 4). Por tanto, a medida que aumenta la fortaleza de un vínculo entre dos individuos, se incrementa la tendencia a confiar en el otro.

En resumen, el riesgo de traición (no ser digno de la confianza depositada en uno) es menor cuando el vínculo entre dos individuos es fuerte (por ejemplo, entre dos amigos) que cuando el vínculo es débil (por ejemplo, entre dos meros conocidos). Sin embargo, el riesgo de traición entre dos individuos es aún menor si existen amigos comunes (figura 5). A medida que aumenta el número de amigos comunes, se incrementan las probabilidades subjetivas de confiar en el otro, y, por tanto, crece la tendencia a confiar. Engañar a un amigo puede generar un sentimiento de culpa enorme, incluso aunque la traición no sea descubierta; pero la pesadumbre puede ser aún mayor si la traición

Figura 5. Amigo común

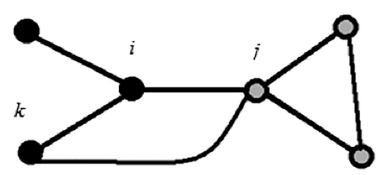

Fuente: elaboración propia 
Figura 6. Red cerrada

Fuente: elaboración propia

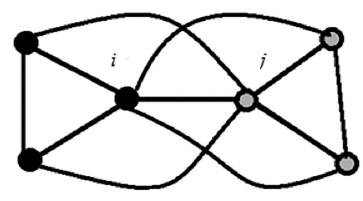

es descubierta por la víctima, y puede ser realmente insoportable si los amigos comunes se enteran de dicha traición (Herreros, 2002, 2004).

¿Por qué aumenta la probabilidad subjetiva de confiar en el otro cuando se incrementa el número de amigos comunes? El mecanismo podría ser el siguiente: el mal comportamiento de un individuo dentro de una red puede afectar gravemente a su reputación (lo digno de confianza que uno sea) entre los miembros de dicha red (Buskens, 1998, 2002; Herreros, 2002, 2004). Así pues, el riesgo de traición en redes cerradas (donde existen muchos amigos comunes) (figura 6) es menor, y, por tanto, la tendencia a confiar en el otro es mayor que en redes abiertas (donde existen pocos amigos comunes).

Dentro de una red cerrada el comportamiento público de cualquier individuo puede ser conocido por el resto de los individuos. Por tanto, cuanto más cerrada sea una red, más probable es que las traiciones sean detectadas y castigadas. Asimismo, el mal comportamiento de un individuo puede influir en su reputación. Por consiguiente, los individuos de una red cerrada poseen más tendencia a confiar que los individuos de una red abierta. Es decir, el incentivo de la reputación reduce la probabilidad de traición y, por tanto, aumenta la tendencia a confiar. Por idénticos motivos, en redes cerradas la gente trabaja más eficazmente que en redes abiertas. Asimismo, los equipos de trabajo constituidos por individuos unidos entre sí finalizan las tareas más rápidamente, con más calidad y menor coste que la mera suma de individuos (Burt, 2005). Por ello, el control de las cadenas de mando vertical en las organizaciones tradicionales ha sido sustituido por el control de los vínculos entre individuos en las organizaciones modernas, lo que fuerza a las personas a no traicionar para conservar e incrementar su reputación dentro del grupo (Lucas, 2013).

La tendencia a confiar entre individuos de una red puede depender, entre otros factores, del número de individuos comunes conectados a dichos individuos, como se ha intentado mostrar. También se sabe que el coeficiente de conglomerado medio de una red es una medida de la conectividad entre los individuos conectados a cada uno de los individuos de dicha red. Por tanto, la tendencia a confiar entre individuos de una red dependería del coeficiente de conglomerado medio de dicha red. Así pues, el coeficiente de conglomerado puede ser considerado como un indicador de la tendencia a confiar.

$$
T=f(\bar{C})
$$


Además, como dicho coeficiente de conglomerado medio depende de las propiedades estructurales de la red, entonces la tendencia a confiar dependerá también de dichas propiedades. Así pues, no es extraño considerar e hipotetizar que diferentes estructuras de redes sociales puedan poseer distintas tendencias a confiar.

Los experimentos virtuales con redes artificiales permiten mantener constantes todos los factores que influyen en el coeficiente de conglomerado medio $y$, por tanto, en la tendencia a confiar, excepto aquel factor que se pretenda analizar cómo influye en tal coeficiente. Así pues, los experimentos virtuales permiten utilizar la cláusula ceteris paribus para observar cómo afectan diversos factores (en particular, en este trabajo, el tipo de estructura de red, la densidad de red, el índice de globalización y el índice de Gini de la distribución de vínculos) en la tendencia a confiar.

\subsection{La tesis de Coleman}

El capital social puede considerarse como un conjunto de recursos insertos en las redes sociales en los que se puede invertir cuando los actores desean aumentar la probabilidad de éxito en sus acciones (Bourdieu, 1986; Coleman, 1990; Lin, 2001). Dos elementos claves del capital social son la información que recorre la estructura de las redes sociales y la confianza mutua entre los actores pertenecientes a la misma estructura de red. Los sujetos no pueden acceder a dichos recursos de capital social si no participan de alguna manera en redes sociales. Así pues, un individuo aislado, que no participe en ninguna red social, no podrá disfrutar de los recursos del capital social (García-Valdecasas, 2011b). Coleman relaciona, en el contexto educativo, la confianza en los jóvenes con una menor deserción escolar y, en general, con un mayor logro educativo (existen algunos críticos de la tesis de Coleman, véase el trabajo de Ramírez y Hernández, 2012). Esta tesis se puede explicitar utilizando términos anclados en el análisis de redes: los jóvenes que viven en redes densas y cerradas tienen menos posibilidad de abandonar el colegio que los que viven en redes poco densas y abiertas. Coleman muestra que los estudiantes que conviven con sus padres y que tienen pocos hermanos poseen menos probabilidades de dejar los estudios que los que conviven en familias monoparentales o que tienen muchos hermanos. Los padres que viven juntos son más eficientes en la vigilancia de los jóvenes que los padres que viven separados. Además, según este autor, los estudiantes que han vivido durante toda la vida en el mismo barrio son menos proclives a dejar la escuela que los que cambian repetidamente de vecindario. Así pues, no es de extrañar que si los padres, los profesores y los vecinos residen en el mismo barrio de siempre, probablemente se conozcan, puedan colaborar juntos y mejorar el control y la vigilancia de los estudiantes. Es decir, al aumentar la densidad de red (redes densas) y el coeficiente de conglomerado (redes cerradas), crece el control y la vigilancia sobre los jóvenes y, por tanto, existen menos posibilidades de que dichos jóvenes abandonen los estudios. Con otras palabras, se incrementa la tendencia a confiar en los jóvenes. 


\section{Redes artificiales}

Las redes artificiales utilizadas en los experimentos virtuales han sido creadas mediante el entorno de programación NetLogo (García y Sancho, 2016; García-Valdecasas, 2016; Wilensky, 1999) siguiendo el modelo de Watts y Strogatz (1998). Dichas redes artificiales están constituidas por un número $n$ de nodos distribuidos a lo largo de un círculo y un número $m$ de vínculos. Inicialmente, cada nodo se une con los nodos de su vecindario (los nodos cercanos). La función de distribución del número de vínculos de los nodos sigue una ley de potencias (power-law), $p(k)=C k^{-y}$, donde la probabilidad, $p(k)$, de que un nodo de la red elegido al azar tenga $k$ vínculos es proporcional a $k^{-y}$, siendo $\gamma$ una constante que varía en función del tipo de red, aunque para la mayoría de las redes sociales se cumple que $2<\gamma<3$, y $C$ es una constante de proporcionalidad asociada a la normalización (Barabási, 2002). En esta ley de potencias unos pocos nodos (llamados hubs) poseen relativamente muchos vínculos, y numerosos nodos tienen relativamente pocos vínculos. Esta distribución estadística es frecuente no solo en las redes sociales, sino también en otros fenómenos. Por ejemplo, Wilfredo Pareto (1906) señaló que el 20\% de la población posee el $80 \%$ de la riqueza y, por el contrario, el $80 \%$ de la población tiene solo el $20 \%$ de la riqueza (la regla del $80-20$ ). Asimismo, George Zipf (1949) descubrió que cuando se escribe se usa mucho un grupo reducido de palabras, pero hay una gran mayoría de ellas que se emplean poco (la ley Zipf). La asignación del número de vínculos a cada nodo se establece fijando un indice de Gini de la distribución del número de vínculos de los nodos, $G$, pero de manera que todos los nodos tengan al menos un vínculo. Se debe señalar que este procedimiento de asignación es de elevada complejidad técnica. A continuación se reemplazan para cada nodo, con una probabilidad $\beta$, los vínculos de corto alcance con nodos del vecindario por vínculos de largo alcance con nodos situados más allá del vecindario. En resumen, se pueden fijar cuatro parámetros en las redes artificiales: $n, m, G$ y $\beta$. Posteriormente, se calcula la densidad de red $(D)$ y el coeficiente de conglomerado medio de red $(\bar{C})$.

El índice de Gini $(G)$ es utilizado en estos experimentos como una medida del grado de desigualdad en la distribución de vínculos entre los nodos de una red (Allison, 1978). Se puede calcular haciendo uso de la siguiente fórmula:

$$
G=\frac{\frac{1}{n^{2}} \sum_{i=1}^{n} \sum_{j=1}^{n}\left|l_{i}-l_{j}\right|}{2 \mu},
$$

donde $\mu$ es la media de la distribución del número de vínculos de los nodos de una red, $n$ es el número total de nodos de la red y $l_{i}$ es el grado modal del nodo $i$, esto es, el número de vínculos del nodo $i$.

De acuerdo con Coleman (1988) y Lin (2001), dicho índice de Gini puede estar relacionado con la desigualdad social. En esta investigación se distingue entre redes igualitarias $(G=0)$ y redes desigualitarias $(G>0)$. 
El parámetro $\beta$ se puede interpretar como un índice de globalización de los vínculos de una red (García-Valdecasas, 2014a). Si $\beta=0$, ningún vínculo con el vecindario es sustituido, la red tiene una estructura regular y todos los vínculos son locales; si $\beta=1$, todos los vínculos con el vecindario son sustituidos, la red posee una estructura aleatoria y todos los vínculos son globales, y si $0<\beta$ $<1$, algunos vínculos con los nodos vecinos son sustituidos, la red presenta una estructura de mundo pequeño (Milgram, 1967; Watts, 1999a, 1999b, 2003, 2004; Watts y Strogatz, 1998) y existe una combinación de vínculos locales y globales. Así pues, al variar $\beta$ se modifica la estructura de la red.

De lo dicho anteriormente se sigue que la estructura de mundo pequeño $(0<\beta<1)$ se puede concebir como una estructura intermedia entre las redes regulares $(\beta=0)$ y las aleatorias $(\beta=1)$. Se dice que una red social, independientemente de su tamaño, tiene una estructura de mundo pequeño cuando existen relativamente pocos pasos de separación por término medio entre dos nodos cualesquiera de dicha red. Conviene también resaltar que la estructura de mundo pequeño está presente en muchas redes sociales reales y por esa razón se utiliza en dicha investigación. Como botón de muestra, véase una estructura de mundo pequeño en la figura 7.

Las redes artificiales deben parecerse en algún sentido a las redes reales, es decir, las primeras deben representar adecuadamente a las segundas para que las conclusiones que se extraigan de las redes artificiales se puedan aplicar a las redes reales. En este sentido, las redes artificiales creadas en este trabajo poseen dos de las características más importantes de las redes reales: la estructura de mundo pequeño y la ley de potencia en la distribución de vínculos (Watts, 2003; Barabási, 2002).

Por último, desde un punto de vista técnico, estas redes artificiales están representadas por grafos simples y no dirigidos. Los grafos simples son aquellos donde no existe más de un vínculo entre dos nodos cualesquiera y tampoco existen vínculos reflexivos de los nodos consigo mismos (no existen bucles). Asimismo, los grafos no dirigidos están constituidos por vínculos no orientados, es decir, vínculos de doble dirección. Un ejemplo de grafo dirigido es la red de tuberías de una ciudad, ya que por las tuberías el agua corre en una única dirección; por el contrario, un ejemplo de grafo no dirigido es la red de

Figura 7. Estructura de mundo pequeño $(n=20 ; m=40 ; G=0,175 ; \beta=0,5)(\bar{C}=0,22 ; D=0,21)$

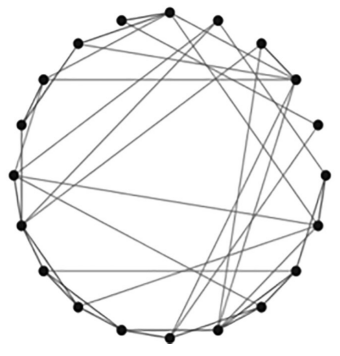


carreteras de un país, porque por una carretera se puede circular en las dos direcciones. El que las redes artificiales sean representadas por grafos simples y no dirigidos simplifica mucho el diseño y la construcción de dicha redes, pero puede reducir el alcance de los resultados de estos experimentos virtuales.

\section{Experimentos virtuales}

Se han llevado a cabo cuatro tipos distintos de experimentos virtuales con redes artificiales y se ha ejecutado 100 veces cada uno de ellos para obtener los resultados medios. El espacio paramétrico de cada experimento viene especificado debajo del título del gráfico correspondiente. Lo esencial de estos experimentos no son los resultados numéricos concretos, sino la forma geométrica de las funciones — que muestran los gráficos—, que se mantiene constante e independiente de los valores de los parámetros iniciales de los experimentos virtuales.

La primera tanda de experimentos virtuales analiza cómo la densidad $(D)$ influye en el coeficiente de conglomerado $(\bar{C})$ y, por consiguiente, en la tendencia a confiar en distintas estructuras de red (caracterizadas por diferentes valores de $\beta$ ) y para un valor nulo del índice de Gini $G=0$. Como muestra el gráfico 1 , para mundos pequeños e igualitarios $(0<\beta<1$ y $G=0)$, así como para redes globales (aleatorias) e igualitarias $(\beta=1$ y $G=0)$, a medida que se incrementa $D$, aumenta $(\bar{C})$, y, por tanto, crece la tendencia a confiar en el interior de dichas redes. Pero para redes locales (regulares) e igualitarias ( $\beta$ = 0 y $G=0$ ), a medida que aumenta $D$, primero disminuye $\bar{C}$, pero después se incrementa, $y$, de forma paralela, primero se reduce la tendencia a confiar,

Gráfico 1. Efecto de $D$ sobre $\bar{C}$ para diferentes valores de $\beta$ y $G=0$. $(n=100 ; m=200,400$, $600 . . ., 1200 ; G=0 ; \beta=0,0,5,1)$

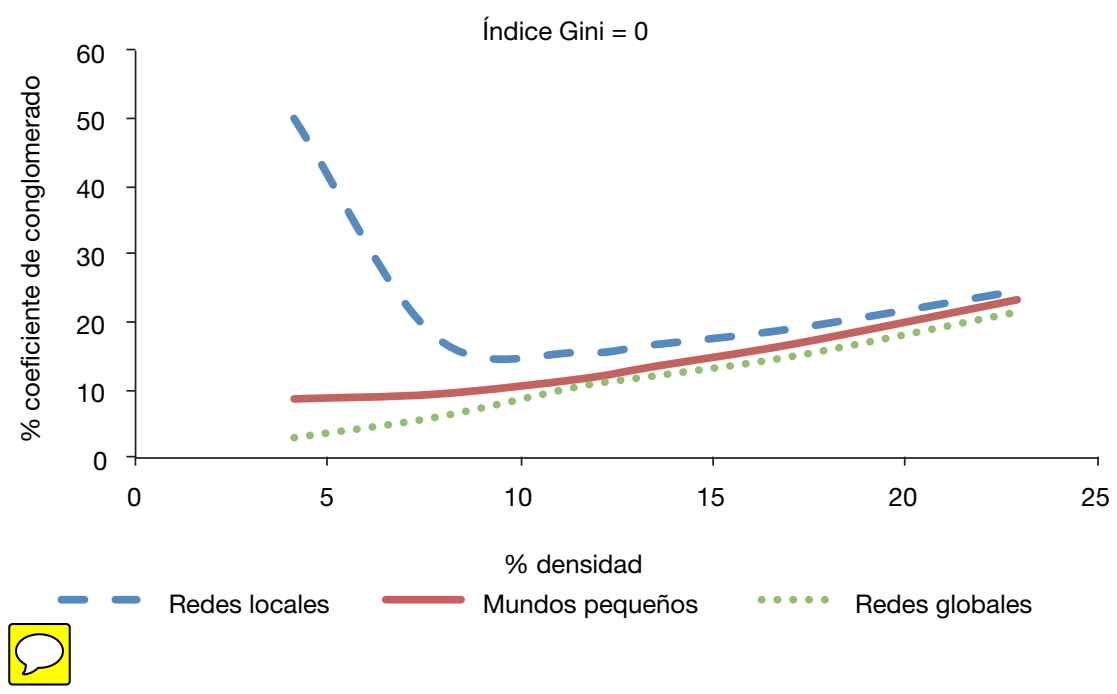


Gráfico 2. Efecto de $D$ sobre $\bar{C}$ para diferentes valores de $\beta$ y $G=0,5$. $(n=100 ; m=200$, $400,600 \ldots, 1200 ; G=0,5 ; \beta=0,0,5,1)$

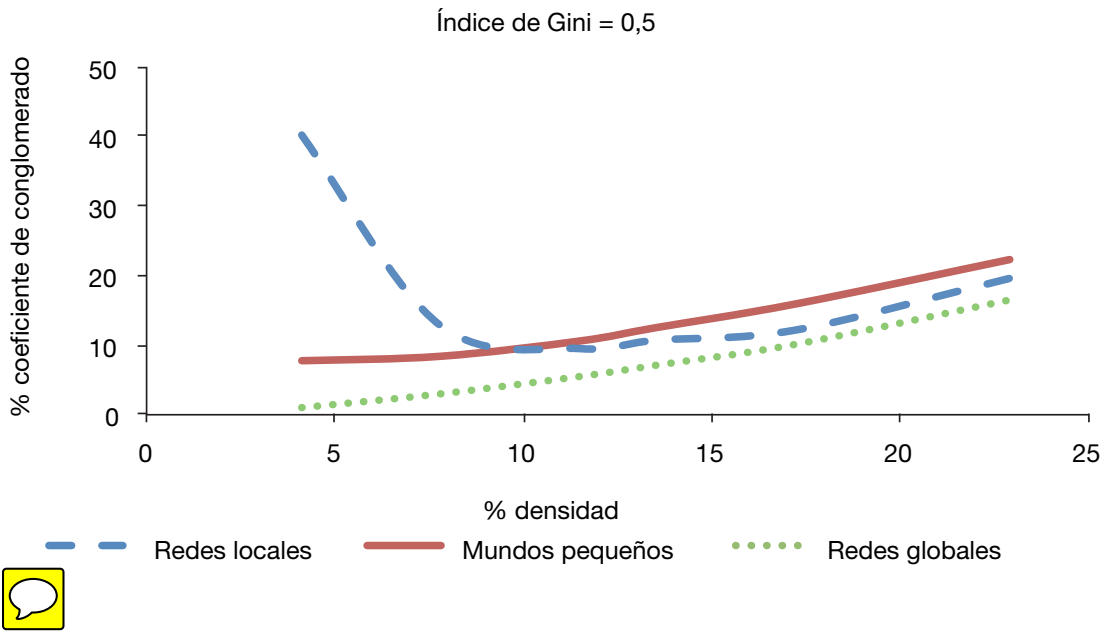

pero después aumenta. Además, para cualquier valor de la densidad, en las redes locales $(\beta=0)$ se confía más que en los mundos pequeños $(0<\beta<1)$, $y$, a su vez, en los mundos pequeños se confía más que en las redes aleatorias $(\beta=1)$. Por otro lado, para un mismo nivel de confianza, las redes locales requieren menos densidad y, por tanto, son de menor coste que los mundos pequeños y las redes globales. En resumen, estos experimentos muestran la importancia de la estructura de red y de la densidad en la tendencia a confiar. Es importante notar que el valor de $n$ y los valores de $m$ (relacionado con la densidad) son arbitrarios. Otros valores de dichos parámetros conducen a resultados muy similares. Asimismo, el valor de $\beta=0,5$ para mundos pequeños es también arbitrario, pero diferentes valores de $\beta=[0,1]$ llevan a resultados equivalentes.

Los resultados del experimento anterior parecen bastante obvios. Sin embargo, si aumenta el valor de $G$, los resultados pueden cambiar significativamente (como muestra el gráfico 2). En la segunda serie de experimentos se muestra que, cuando aumenta $G$, en los mundos pequeños y desigualitarios $(0<\beta<1$ y $G=0,5)$ aumenta la tendencia a confiar más que en las redes locales y desigualitarias ( $\beta=0$ y $G=0,5)$ y que en las redes globales y desigualitarias $(\beta=1$ y $G=0,5)$ a partir de cierto valor de la densidad. En este caso concreto, si las tres redes poseen los mismos niveles de confianza, los mundos pequeños son de menor coste que las redes locales y las redes globales, porque requieren menos densidad de red para su mantenimiento. Probablemente los mundos pequeños hayan sobrevivido en la historia de la humanidad (Watts, 2003) porque han aumentado la tendencia a confiar y, por tanto, refuerzan la cooperación, cuestión clave para la supervivencia de nuestra especie, frente a otras estructuras de red en sociedades con desigualdades crecientes. Así, estos 
Gráfico 3. Efecto de $\beta$ sobre $\bar{C}$ para $D=0,15,0,20,0,25$ y $G=0,5(n=100 ; m=742,990$, 1238; $G=0,5 ; \beta=0,0,1,0,2 \ldots, 1)$

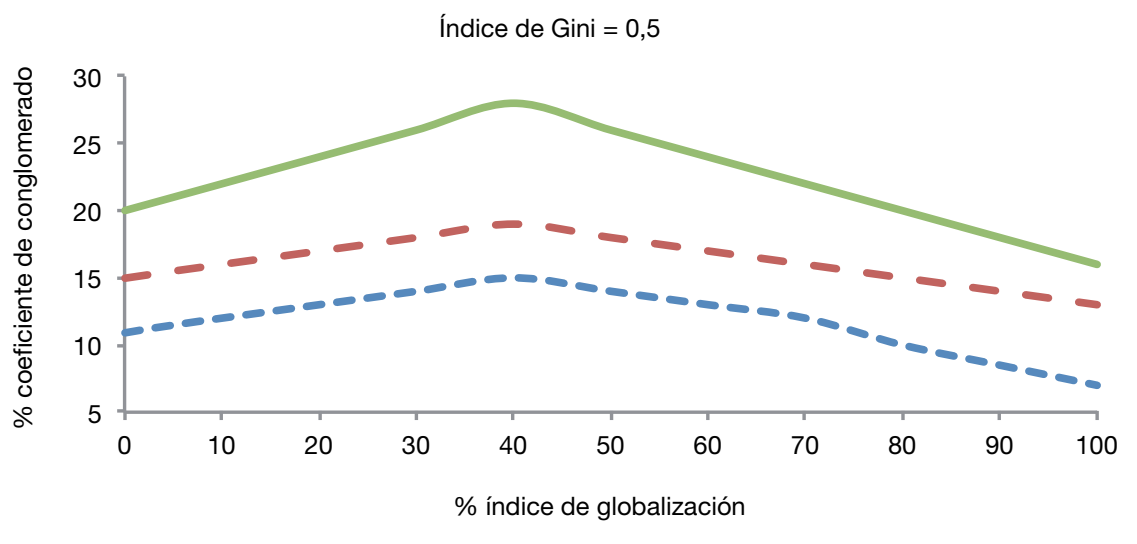

2

$$
D=15 \%-D=20 \% \longrightarrow D=25 \%
$$

experimentos muestran la relevancia de los mundos pequeños en la tendencia a confiar en sociedades desigualitarias.

La tercera tanda de experimentos analiza cómo influye el índice de globalización $(\beta)$ en el coeficiente de conglomerado $(\bar{C}) \mathrm{y}$, por consiguiente, en la tendencia a confiar, en redes desigualitarias (por ejemplo, $G=0,5$ ) para varias densidades de red dadas $(D=0,15,0,2,0,25)$. Otros valores de $G$ y $D$ arrojan resultados similares. El rango elegido (desde $D=15 \%$ hasta $D=25 \%$ ) muestra con mayor nitidez las diferencias en el coeficiente de conglomerado. El gráfico 3 enseña que existe un valor de $\beta(\approx 0,4)$ para el cual el valor de $(\bar{C})$ es máximo y, por tanto, la tendencia a confiar es máxima. Así pues, existe un valor de $\beta$ óptimo para la tendencia a confiar. Por tanto, los seres humanos de sociedades cerradas formadas por redes locales $(\beta=0)$ y de sociedades abiertas constituidas por redes globales $(\beta=1)$ confían menos que los individuos de sociedades constituidas por mundos pequeños $(\beta \approx 0,4)$, pero solo si dichas sociedades son desigualitarias. También se puede observar que, a medida que aumenta la densidad de red, se incrementa la tendencia a confiar.

La cuarta serie de experimentos estudia el efecto del índice de Gini $(G)$ sobre el coeficiente de conglomerado $(\bar{C})$ y, por tanto, sobre la tendencia a confiar en mundos pequeños (por ejemplo, $\beta=0,5$ ) para varias densidades de red dadas $(D=0,15,0,2,0,25)$. Otros valores de $D$ y $\beta$ generan resultados parecidos. El gráfico 4 muestra que, a medida que aumenta $G$, disminuye $(\bar{C})$ y, por consiguiente, se reduce la tendencia a confiar. Es decir, cuando se incrementan las desigualdades sociales y el resto de los parámetros permanecen constantes, disminuye la tendencia a confiar. También se puede observar que, cuando se incrementa la densidad de red, aumenta la tendencia a confiar. 
Gráfico 4. Efecto de $G$ sobre $\bar{C}$ para $D=0,15,0,20,0,25$ y $\beta=0,5(n=100 ; m=742,990$, 1238; $G=0,0,1,0,2 \ldots 0,9 ; \beta=0,5)$

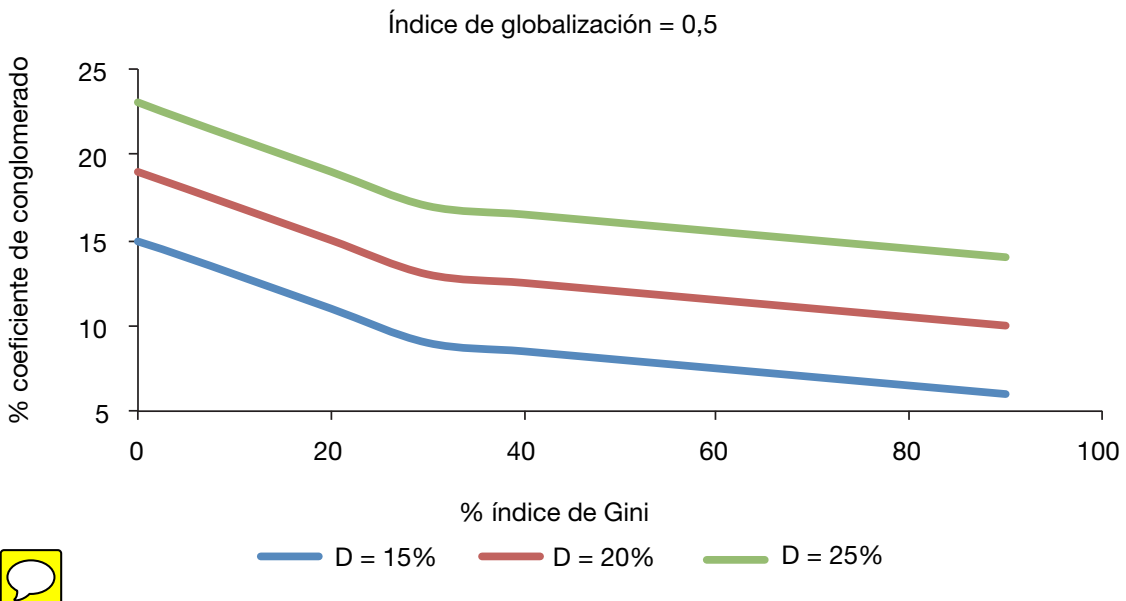

\section{Discusión y conclusiones}

Los resultados de estos experimentos virtuales con redes artificiales ponen de manifiesto la relevancia del tipo de estructura de red y de ciertas propiedades estructurales, a saber, la densidad, el índice de globalización y el índice de Gini sobre el coeficiente de conglomerado, y, por tanto, sobre la tendencia a confiar, y, por consiguiente, sobre la cooperación entre los agentes de una red. Los resultados se pueden resumir de la siguiente forma:

a) Para redes igualitarias $(G=0)$, las redes locales $(\beta=0)$ son las que poseen más tendencia a confiar.

b) Para redes desigualitarias $(G>0)$, a partir de cierta densidad de red, los mundos pequeños $(0<\beta<1)$ son los que tienen más tendencia a confiar.

c) En casi todos los casos, al aumentar la densidad se incrementa la tendencia a confiar, aunque este resultado se puede derivar directamente de la definición del coeficiente de conglomerado.

d) Existe un índice de globalización óptimo para la tendencia a confiar, es decir, existe un valor de dicho índice para el cual la tendencia a confiar es máxima. Así pues, se puede concluir que los mundos pequeños son las estructuras óptimas para la tendencia a confiar en redes desigualitarias, $y$, dentro de dichos mundos, existe un valor óptimo de $\beta$ que maximiza la tendencia a confiar.

e) A medida que crece el índice de Gini de la distribución de vínculos, se reduce la tendencia a confiar, esto es, cuando la desigualdad social aumenta, la tendencia a confiar disminuye.

f) Por último, para mundos pequeños e igualitarios $(0<\beta<1$ y $G=0)$, así como para redes globales (aleatorias) e igualitarias ( $\beta=1$ y $G=0)$, a 
medida que se incrementa $D$, aumenta $\bar{C}$ y, por tanto, crece la tendencia a confiar en el interior de dichas redes. Pero para redes locales (regulares) e igualitarias ( $\beta=0$ y $G=0$ ), a medida que aumenta $D$, primero disminuye $\vec{C}$, pero después se incrementa y, de forma paralela, primero se reduce la tendencia a confiar, pero después aumenta. Este conjunto de resultados, junto a otros muchos (Barabási, 2002; Burt, 2004, 2005; Coleman et al., 1957; Hedström, 2005; Hedström y Bearman, 2009; Granovetter, 1973, 1974; Milgram, 1967; Newman, 2003; Newman et al., 2003; Schelling, 1978; Watts, 2003), apoya la tesis de la pertinencia de la estructura de las redes sociales y sus propiedades para explicar los procesos dinámicos que acontecen en su interior.

Estos resultados permiten también evaluar la tesis de Coleman sobre la importancia de las redes densas y cerradas en la tendencia a confiar en que los jóvenes no abandonen los estudios. La tesis de Coleman, explicada en términos del análisis de redes, diría que, a medida que aumenta la densidad de red, se incrementa el coeficiente de conglomerado y, por tanto, crece el control y la vigilancia sobre los jóvenes, lo que se traduce en una reducción de la posibilidad de abandonar los estudios. Los experimentos virtuales realizados en este trabajo ponen de manifiesto también otra perspectiva diferente. Se puede elevar el control y la vigilancia de los jóvenes sin tener necesariamente que aumentar la densidad de red, esto es, sin aumentar el coste en el mantenimiento de vínculos, ya que se puede incrementar dicho control y dicha vigilancia variando la estructura de red (caracterizada por $\beta$ ) o modificando el grado de desigualdad en la distribución de vínculos $(G)$. Por tanto, estos experimentos no señalan que la tesis de Coleman sea falsa, sino que no llega a explorar del todo la compleja relación entre la estructura de las redes sociales y el control y la vigilancia de los jóvenes. Con otras palabras, los resultados de estos experimentos muestran que lo relevante para la tendencia a confiar no es solo cuántos agentes están vinculados entre sí (la densidad de red), sino también cómo se distribuyen las conexiones entre los agentes de una red (el índice de globalización y el índice de Gini de la distribución de vínculos).

En este trabajo se ha analizado cómo ciertas propiedades estructurales de las redes (con gran significado sociológico) influyen en la tendencia a confiar entre los individuos que forman dichas redes, pero también se podría estudiar el impacto de otros parámetros estructurales. Se podría indagar, por ejemplo, cómo ciertas propiedades, tales como el grado de intermediación, el grado de cercanía, el diámetro de red, los K-grupos, etc., afectan a la tendencia a confiar $y$, por consiguiente, a la cooperación entre los miembros de una red.

Se debe indicar, por último, que los experimentos computacionales realizados en este trabajo poseen algunos inconvenientes importantes: primero, la comprobación empírica de sus resultados es muy difícil, y si dichos resultados no se pueden comprobar empíricamente, entonces no hay manera de conocer su pertinencia (García-Valdecasas, 2013, 2014a; Gilbert, 2008; GonzálezBailón, 2004). Segundo, la limitada posibilidad de que otros investigadores 
puedan reproducir dichos experimentos para comprobar si los resultados son consistentes (Gilbert, 2008), puesto que, como ocurre con cualquier experimento científico, es necesario comprobar si los resultados de la simulación se deben a causas substantivas o a defectos en el diseño y la programación de las redes artificiales (González-Bailón, 2004). Es posible que distintos diseños y programas realizados por diferentes grupos de investigación obtengan resultados divergentes sobre el mismo fenómeno social (García-Valdecasas, 2016).

\section{Referencias bibliográfícas}

Alesina, Alberto y La Ferrara, Eliana (2002). «Who Trusts Others?». Journal of Public Economics, 85, 207-234. <https://doi.org/10.1016/S0047-2727(01)00084-6>

Allison, Paul (1978). «Measures of Inequality». American Sociological Review, 43, 865-880. $<$ https://doi.org/10.2307/2094626>

Axelrod, Robert (1984). The Evolution of Cooperation. Nueva York: Basic Books. $<$ https://doi.org/10.1007/978-3-531-90400-9_7>

- (1997). «Advancing the Art of Simulation in the Social Science». En: Conte, Rosaria; Hegselmann, Rainer y Terna, Pietro (eds.). Simulation Social Phenomena. Berlín: Springer-Verlag. <https://doi.org/10.1007/978-3-662-03366-1_2>

BACK, István y FLACHE, Andreas (2006). «The Viability of Cooperation Based on Interpersonal Commitment». Journal of Artificial Societies and Social Simulation, 9(1). Recuperado el 21 de mayo de 2017, de <http://jasss.soc.surrey.ac.uk/9/1/12.html>.

Barabási, Albert-László (2002). Linked: The New Science of Networks. Cambridge, MA: Perseus. $<$ https://doi.org/10.2307/20033300>

Boccaletti, Stefano et al. (2006). "Complex networks: Structure and dynamics». Physics Reports, 424, 175-308. $<$ https://doi.org/10.1016/j.physrep.2005.10.009>

Bourdieu, Pierre (1986). «The forms of Capital». En: Richardson, J.G. (ed.). Handbook of theory and research for the sociology of education. Nueva York: Greenwood. $<$ https://doi.org/10.1002/9780470755679.ch15>

Burt, Ronald S. (2004). «Structural holes and good ideas». American Journal of Sociology, 110, 349-399. <https://doi.org/10.1086/421787>

- (2005). Brokerage and Closure. Oxford: Oxford University Press. <https://doi.org/10.1007/978-3-658-21742-6_23>

Burt, Ronald S.; Bian, Yanjie y Opper, Sonja (2018). «More or less guanxi: Trust is 60\% network context, $10 \%$ individual difference». Social Networks, 54, 12-25. <https://doi.org/10.1016/j.socnet.2017.12.001>

Buskens, Vincent W. (1998). «The social Structure of Trust». Social Networks, 20, 265-289. <https://doi.org/10.1016/s0378-8733(98)00005-7>

- (2002). Social networks and trust. Boston, Dordrecht y Londres: Kluwer Academic Publishers. <https://doi.org/10.1007/b109038> 
Carrington, Peter J.; Scott, John y Wasserman, Stan (2005). Models and Methods in Social Network Analysis. Cambridge: Cambridge University Press. <https://doi.org/10.1017/CBO9780511811395>

Chow, Wing S. y Chan, Lai S. (2008). «Social network, social trust and shared goals in organizational knowledge sharing». Information \& Management, 45(7), 458-465. <https://doi.org/10.1016/j.im.2008.06.007>

Coleman, James S. (1988). «Social Capital in the Create of Human Capital». American Journal of Sociology, 94, 95-120. <https://doi.org/10.1086/228943>

- (1990). Foundations of Social Theory. Cambridge: Harvard University Press.

Coleman, James S.; Menzel, Herbert y Katz, Elihu (1957). "The diffusion of an innovation among physicians». Sociometry, 20, 253-270. <https://doi.org/10.2307/2785979>

Cook, Karen S. y Gerbasi, Alexandra (2009). «Trust». En: Hedström, Peter y Bearman, Peter (eds.). The Oxford Handbook of Analytical Sociology. Oxford: Oxford University Press. <https://doi.org/10.1093/oxfordhb/9780199215362.013.10>

Cruz Gómez, Irene y Verd, Joan M. (2013). «The strength of ties: A theoretical and empirical exploration of its multiple meanings». Empiria: Revista de Metodología de Ciencias Sociales, 26, 149-174. <https://doi.org/10.5944/empiria.26.2013.7156>

Diestel, Reinhard (2000). Graph Theory. Nueva York. <https://doi.org/10.1002/1097-0118(200012)35:4<273::AID-JGT4>3.0.CO;2-B>

Dunn, John (1993). «Trust». En: Goodin, Robert E. y Pettit, Philip (eds.). A Companion to Contemporary Political Philosophy. Oxford: Blackwell. <https://doi.org/10.1111/b.9780631199519.1995.x>

Epstein, Joshua M. (2006). Generative Social Science: Studies in Agent-Based Computational Modelling. Princeton: Princeton University Press. <https://doi.org/10.1515/9781400842872>

Funuyama, Francis (1995). Trust: The Social Virtues and the Creation of Prosperity. Nueva York: Free Press.

García-Valdecasas, José Ignacio (2011a). «La simulación basada en agentes: Una nueva forma de explorar los fenómenos sociales». Revista Española de Investigaciones Sociológicas (REIS), 136, 91-110. <https://doi.org/10.5477/cis/reis.136.91>

- (2011b). «Una definición estructural de capital social». Redes: Revista Hispana para el Análisis de Redes Sociales, 20(1). $<$ https://doi.org/10.5565/rev/redes.411>

- (2013). «Grupos moderados y tolerantes en la polarización política de las redes sociales». Revista Española de Investigaciones Sociológicas (REIS), 136, 25-44. <https://doi.org/10.5477/cis/reis.142.25>

- (2014a). «El impacto de la estructura de las redes sociales sobre el acceso de los individuos al mercado laboral». Revista Internacional de Sociología (RIS), 72(2), 303-321. <https://doi.org/10.3989/ris.2012.09.13>

- (2014b). «Explicación, mecanismo y simulación: Otra forma de hacer sociología». Empiria: Revista de Metodología de Ciencias Sociales, 28, 35-58. $<$ https://doi.org/10.5944/empiria.28.2014.12120>

- (2015). «La estructura compleja de las redes sociales». Revista Española de Sociología (RES), 24, 65-84. 
- (2016). Simulación basada en agentes: Una introducción a NetLogo. Madrid: Centro de Investigaciones Sociológicas. Cuadernos Metodológicos, 53.

García-VAldecasas, José Ignacio y López, Iván (2017). «Un modelo basado en agentes para el análisis de la segregación étnica espacial urbana». Revista de Geografía Norte Grande, 67, 145-165. <https://doi.org/10.4067/S0718-34022017000200008>

García Vázquez, J.C. y Sancho Caparrini, F. (2016). NetLogo: Una herramienta de Modelado. Payhip.

Gasalla, Jose María y Navarro, Leila (2008). Confianza: La clave para el éxito personal y empresarial. Madrid: Empresa Activa.

Ghavipour, Mina y Meybodi, Mohammad R. (2018). «A dynamic algorithm for stochastic trust propagation in online social networks: Learning automata approach». Computer Communications, 123, 11-23. <https://doi.org/10.1016/j.comcom.2018.04.004>

Gilbert, Nigel (2008). Agent-Based Models: Quantitative applications in the social science 153. Londres: Sage. <https://doi.org/10.4135/9781412983259>

Gilbert, Nigel у Аввотт, Andrew (2005). «Social Science Computation». American Journal of Sociology, 110, 859-863. <https://doi.org/10.1086/430413>

Gilbert, Nigel y Troitzsch, Klaus G. (2005). Simulation for the Social Scientist. Milton Keynes: Open University Press.

GonZÁLEZ-BAIlón, Sandra (2004). "¿Sociedades artificiales?: Una introducción a la simulación social». Revista Internacional de Sociología, 39, 199-222. <https://doi.org/10.3989/ris.2004.i39.268>

- (2006). «The Role of Dynamic Network in Social Capital: A Simulation Experiment». Papers: Revista de Sociología, 80, 171-194. <https://doi.org/10.5565/rev/papers/v80n0.1774>

Granovetter, Mark (1973). «The strength of weak ties». American Journal of Sociology, 76, 1360-1380. <https://doi.org/10.1086/225469>

- (1974). Getting a Job: A Study of Contacts and Careers. Cambridge: Harvard University Press.

Hanneman, Robert A. y Riddle, Mark (2005). Introduction to social network methods. Riverside, CA: University of California, Riverside.

Hardin, Russell (2006). Trust. Cambridge: Polity.

Hedström, Peter (2005). Dissecting the Social: On the Principles of Analytical Sociology. Cambridge: Cambridge University Press. <https://doi.org/10.1017/CBO9780511488801>

Hedström, Peter y Bearman, Peter (eds.) (2009). The Oxford Handbook of Analytical Sociology. Oxford: Oxford University Press. <https://doi.org/10.1093/oxfordhb/9780199215362.001.0001>

Herreros, Francisco (2002). ¿Por qué confiar?: El problema de la creación de capital social. Madrid: CEACS.

- (2003). «Las fuentes de la confianza social». Revista Internacional de Sociología, 35, 151-175. <https://doi.org/10.2307/3541411>

- (2004). The Problem of Forming Social Capital: Why Trust? Nueva York y Londres: Palgrave. <https://doi.org/10.1057/9781403978806> 
LewIn, Kurt (1936). Principles of Topological Psychology. Nueva York, NY: Harper \& Row.

- (1951). Field Theory in the Social Sciences. Nueva York, NY: Harper.

Lin, Nan (2001). Social Capital: A Theory of Social Structure and Action. Cambridge: Cambridge University Press.

<https://doi.org/10.1017/CBO9780511815447>

Lucas, Antonio (ed.) (2013). Sociología de las organizaciones. Madrid: Fragua.

Macy, Michael W. y Flache, Andreas (2009). "Social Dynamics from the Bottom Up: Agent-Based Model of Social Interaction». En: Hedström, Peter y BeARman, Peter (eds.). The Oxford Handbook of Analytical Sociology. Oxford: Oxford University Press.

<https://doi.org/10.1093/oxfordhb/9780199215362.013.11>

Macy, Michael W. y Miller, Robert (2002). «From Factors to Actors: Computational Sociology and Agent-Based Modelling». Annual Review of Sociology, 28, 143-66. <https://doi.org/10.1146/annurev.soc.28.110601.141117>

Milgram, Stanley (1967). «The Small World Problem». Psychology Today, 2, 60-67. <https://doi.org/10.1037/e400002009-005>

Molina, José Luis (2001). El análisis de las redes sociales: Una introducción. Barcelona: Bellaterra.

Montero, José; Zmerli, Sonja y Newton, Ken (2008). «Confianza social, confianza política y satisfacción con la democracia». Revista Española de Investigaciones Sociológicas, 138, 11-54. <https://doi.org/10.2307/40184879>

Moreno, Jacob L. (1934). Who shall survive?: A new approach to the problem of human interrelations. Nueva York, NY: Beacon Press.

Newman, Mark E.J. (2003). "The Structure and Function of Complex Networks». SIAM Review, 45, 167-256. <https://doi.org/10.1137/S003614450342480>

Newman, Mark E.J.; Barabási, Albert-László y Watts, Duncan J. (2003). The Structure and Dynamics of Networks. Princeton: Princeton University Press. <https://doi.org/10.1007/springerreference_60528>

Paramio, Ludolfo (1999). "Estrategias partidarias y desconfianza política». Revista Internacional de Sociología, 22, 183-201.

Pareto, Vilfredo (1906). Manual of political economy. Oxford: Oxford University Press, 2014.

Putnam, Robert D. (1993). «The Prosperous Community: Social Capital and Public Life». The American Prospect, 13, 35-42.

- (1995). «Bowling Alone: America's Declining Social Capital». Journal of Democracy, 6, 65-78. <https://doi.org/10.1353/jod.1995.0002>

- (2000). Bowling Alone: The collapse and revival of American community. Nueva York: Simon and Schuster. <https://doi.org/10.1145/358916.361990>

Ramírez, Jorge y Hernández, Eduardo (2012). "¿Tenía razón Coleman?: Acerca de la relación entre capital social y logro educativo». Sinéctica: Revista Electrónica de Educación, 39, 1-14.

Requena, Félix (1989). «El concepto de red social». Revista Española de Investigaciones Sociológicas, 48, 137-152. <https://doi.org/10.2307/40183465> 
Rodríguez, Josep A. (1995). Análisis estructural y de redes. Madrid: Centro de Investigaciones Sociológicas.

Rousseau, Denise M.; Sitkin, Sim B.; Burt, Ronald S. y Camerer, Colin (1998). "Not so different after all: A cross-discipline view of trust». Academy of Management Review, 23(3), 393-404. <https://doi.org/10.5465/amr.1998.926617>

Schelling, Thomas C. (1978). Micromotives and Macrobehavior. Nueva York: W.W. Norton.

Sieger, David A. (2009). «Social Networks and Collective Action». American Journal of Political Science, 53, 122-138. <https://doi.org/10.1111/j.1540-5907.2008.00361.x>

Snijders, Chris (1996). Trust and Commitments. Amsterdam: Thesis Publishers.

Squazzoni, Flaminio (2012). Agent-Based Computational Sociology. Singapur: Wiley. <https://doi.org/10.1002/9781119954200>

Steglich, Christian; Snijders, Tom A.B. y Pearson, Michael (2010). «Dynamic Networks and Behavior: Separating Selection from Influence». Sociological Methodology, 40(1), 329-393. <https://doi.org/10.1111/j.1467-9531.2010.01225.x>

Sztompka, Piotr (2006). «New Perspectives on Trust». American Journal of Sociology, $112,905-919$. <https://doi.org/10.1086/510993>

Wasserman, Stan y Faust, Katherine (1994). Social Network Analysis. Cambridge: Cambridge University Press. <https://doi.org/10.1017/CBO9780511815478>

Watts, Duncan J. (1999a). «Network, Dynamic and Small-World Phenomenon». American Journal of Sociology, 105, 493-527. <https://doi.org/10.1086/210318>

- (1999b). Small Worlds: The Dynamic between Order and Randomness. Princeton: Princeton University Press. $<$ https://doi.org/10.2307/j.ctv36zr5d>

- (2003). Six Degrees: The Science of a Connected Age. Nueva York: Norton. $<$ https://doi.org/10.5860/choice.40-6452>

- (2004). "The new science of networks». Annual Review of Sociology, 30, 243-270. <https://doi.org/10.1146/annurev.soc.30.020404.104342>

Watts, Duncan J. y Strogatz, Steven H. (1998). "Collective dynamics of "small world" networks». Nature, 393, 440-442. <https://doi.org/10.1038/30918>

Wilensky, Uri (1999). NetLogo. Evanston, IL: Northwestern University. Center for Connected Learning and Computer-Based Modeling. Recuperado de <http://ccl. northwestern.edu/netlogo/>.

Wu, Jian y Chiclana, Francisco (2014). «A social network analysis trust-consensus based approach to group decision-making problems with interval-valued fuzzy reciprocal preference relations». Knowledge-Based Systems, 59, 97-107. <https://doi.org/10.1016/j.knosys.2014.01.017>

ZIPF, George (1949). Human Behavior and the Principle of Least Effort: An Introduction to Human Ecology. Cambridge, Mass.: Addison-Wesley Press. <https://doi.org/10.1002/1097-4679(195007)6:3<306::aid-jclp2270060331>3.0. co;2-7> 